\title{
Intraspecific evolution of the intercellular signaling network underlying a robust developmental system
}

\author{
Josselin Milloz, Fabien Duveau, Isabelle Nuez, and Marie-Anne Félix ${ }^{1}$ \\ Institut Jacques Monod, CNRS-University Denis Diderot-Paris 7-UPMC, 75251 Paris cedex 05, France
}

\begin{abstract}
Many biological systems produce an invariant output when faced with stochastic or environmental variation. This robustness of system output to variation affecting the underlying process may allow for "cryptic" genetic evolution within the system without change in output. We studied variation of cell fate patterning of Caenorhabditis elegans vulva precursors, a developmental system that relies on a simple intercellular signaling network and yields an invariant output of cell fates and lineages among C. elegans wild isolates. We first investigated the system's genetic variation in C. elegans by means of genetic tools and cell ablation to break down its buffering mechanisms. We uncovered distinct architectures of quantitative variation along the Ras signaling cascade, including compensatory variation, and differences in cell sensitivity to induction along the anteroposterior axis. In the unperturbed system, we further found variation between isolates in spatio-temporal dynamics of Ras pathway activity, which can explain the phenotypic differences revealed upon perturbation. Finally, the variation mostly affects the signaling pathways in a tissue-specific manner. We thus demonstrate and characterize microevolution of a developmental signaling network. In addition, our results suggest that the vulva genetic screens would have yielded a different mutation spectrum, especially for Wnt pathway mutations, had they been performed in another C. elegans genetic background.
\end{abstract}

[Keywords: Signaling network; evolution; C. elegans; vulva; robustness; cryptic variation]

Supplemental material is available at http://www.genesdev.org.

Received June 30, 2008; revised version accepted August 29, 2008.

A widespread property of biological systems is the robustness of their output to stochastic, environmental, or genetic variation. Empirical studies have deciphered the mechanistic causes of such robustness in a small number of systems (Alon et al. 1999; Eldar et al. 2002, 2003; Gregor et al. 2007). In computational models, environmental or genetic variation may be simulated by altering the parameters describing the dynamical system (Barkai and Leibler 1997; von Dassow et al. 2000; Meir et al. 2002). In principle, robustness to parameter variation may enable system evolution without change in its output (cryptic variation) (Gibson and Wagner 2000; Gibson and Dworkin 2004). Yet, despite an extensive theoretical corpus (for reviews, see de Visser et al. 2003; Flatt 2005), few experimental studies have examined evolution of robust systems. A key question is to understand whether and how the processes underlying robust biological systems evolve. Microevolutionary studies of cryptic genetic variation have so far been limited by the complexity of the trait used or by the lack of detailed molecular knowledge of the system (Gibson and van Helden 1997;

${ }^{1}$ Corresponding author.

E-MAIL felix@ijm.jussieu.fr; FAX 33-1-44-27-52-65.

Article is online at http://www.genesdev.org/cgi/doi/10.1101/gad.495308.
Gibson et al. 1999; Gibson and Dworkin 2004; Zauner and Sommer 2007). We thus decided to study cryptic intraspecific genetic variation in a well-characterized developmental system: vulva cell fate patterning in the nematode Caenorhabditis elegans (Sternberg 2005). This developmental process involves a small number of cells that achieve a precise and invariant cell fate pattern under the control of a well-characterized molecular network. In contrast to previous studies, the single-cell resolution of the system, its extensive description, and the available methods in C. elegans enable detailed investigation of quantitative, spatial, and temporal variation in the underlying cell signaling network.

The vulva is the egg-laying and copulatory organ of $C$. elegans hermaphrodites. It develops from a row of ventral epidermal precursor cells, P3.p to P8.p, which are competent to adopt vulval fates (Sternberg 2005). These cells achieve a spatial fate pattern that is basically invariant within and between all C. elegans wild isolates (Fig. 1A,B). Only three cells (P5.p-P7.p) normally adopt a vulval fate: P6.p adopts an inner (or $1^{\circ}$ ) vulval fate, while P5.p and P7.p adopt lateral (or $2^{\circ}$ ) vulval fates. Under normal conditions, P3.p, P4.p, and P8.p adopt a nonvulval (or $3^{\circ}$ ) fate, yet they are able to replace P5.p-P7.p, and thus belong to the vulval competence group. The spatial 
cell fate pattern $\left(3^{\circ} 3^{\circ} 2^{\circ} 1^{\circ} 2^{\circ} 3^{\circ}\right)$ is organized around the gonadal anchor cell, located close to P6.p, which emits an inductive LIN-3/EGF signal (Sternberg 2005). The LIN-3/EGF inducer can act as a morphogen: High doses induce a $1^{\circ}$ fate, while low doses may induce a $2^{\circ}$ fate (Katz et al. 1995). Upon activation of the EGF/Ras cascade, the P6.p cell triggers a lateral Delta/Notch signaling pathway that simultaneously induces a $2^{\circ}$ fate and inhibits the Ras pathway in P5.p and P7.p (Fig. 1C; Sternberg 2005). The Wnt signaling pathway maintains vulval cell competence in the L2 stage, contributes to the induction of vulval versus $3^{\circ}$ fates and later plays multiple roles in $2^{\circ}$ lineage polarity (Eisenmann et al. 1998; Green et al. 2008). This developmental system with a small number of cells is thus simple and invariant: Schematically, the anchor cell induces P6.p (EGF pathway), which in turn induces its neighbors P5.p and P7.p (Notch pathway). Compared with other developmental systems-for example, those based on long-range gradients for which concentration ranges may be critical-the mechanistic basis of this system's robustness to variation is thus straightforward (Félix and Wagner 2008).

Nematode vulva formation has also been a model of choice for comparative developmental studies. At large evolutionary scales (different families and genera), changes in cell fate patterning mechanism and cell lineage were demonstrated (Félix 1999; Félix et al. 2000;

Figure 1. Uncovering cryptic genetic variation in C. elegans vulva development. (A) C. elegans vulva development. The invariant cell fate pattern $\left(3^{\circ} 3^{\circ} 2^{\circ} 1^{\circ} 2^{\circ} 3^{\circ}\right)$ is established through the interplay of three cell signaling pathways: Egf-Egfr-Ras, DeltaNotch, and Wnt- $\beta$-catenin. (B) Vulval precursor cell lineage. Letters indicate the division plane: (T) transverse (left-right), (L) longitudinal (anteroposterior), (U) undivided. The primary lineage is characterized by division of all four grandaughters in a transverse orientation. The secondary lineage ("LLTU" or "UTLL") is characterized by an absence of division of one granddaughter and adhesion to the cuticle (underline). The tertiary lineage is a nonvulval fate, with a single division ("S", fusion with the epidermal syncytium hyp7). The $3^{\circ} 3^{\circ} 2^{\circ} 1^{\circ} 2^{\circ} 3^{\circ}$ pattern and Pn.p cell lineage are invariant in all wild isolates (except for P3.p division frequency) (Delattre and Félix 2001). (C) Vulva signaling network showing the different Ras pathway components and the interplay between Ras and Notch pathways (see main text for description). (D) Schematic representation of invasive approaches to uncover cryptic genetic variation in the system. The graph shows a theoretical curve of the number of induced Pn.p cells $\left(1^{\circ}\right.$ or $2^{\circ}$ fate) as a function of EGF dose or Ras pathway activity; this number may range from 0 (no anchor cell) to 6 (egf overexpression). The plateau at the wild-type phenotype of three induced cells materializes robustness to some variation in the EGF signal or Ras pathway activity (Braendle et al. 2008; Félix and Wagner 2008). While N2, the reference C. elegans wild isolate, is located on the plateau under standard conditions, a second isolate $(\mathrm{X})$ could be located at a different position on the plateau (stasis in the final fate pattern). Applying the same perturbation to both isolates will schematically shift their respective positions along the $X$-axis, thus uncovering an initially silent difference. As perturbations, we applied mutations in the Ras pathway (Figs. 2, 3) and anchor cell ablations (Fig. 4). (gf) Gain of function; (rf) reduction of function.
Sommer 2005; Kiontke et al. 2007). In contrast, the cell fate pattern and cell lineage are remarkably similar at a smaller scale, within the Caenorhabditis genus. Yet, despite this stasis in the final phenotype, the detailed mechanisms that control vulva development have evolved (Kirouac and Sternberg 2003; Félix 2007). More specifically, mild experimental variations of the pathway activities in C. elegans could mimic the system's behavior in the different species, showing that interspecific differences in the establishment of the vulva precursor fate pattern could be explained by modifications of the relative contributions of the EGF/Ras and Notch pathways (Félix 2007).

Here we study the variation of vulva formation at an even smaller evolutionary scale (i.e., among wild C. elegans isolates), which renders genetic analysis possible. We selected seven independent C. elegans wild isolates that display a large genetic and geographic diversity (Denver et al. 2003; Stewart et al. 2005; Dolgin et al. 2008). Each isolate is an isogenic line, a situation that is representative of wild genotypes (Barrière and Félix 2005, 2007). Importantly, the use of isogenic lines

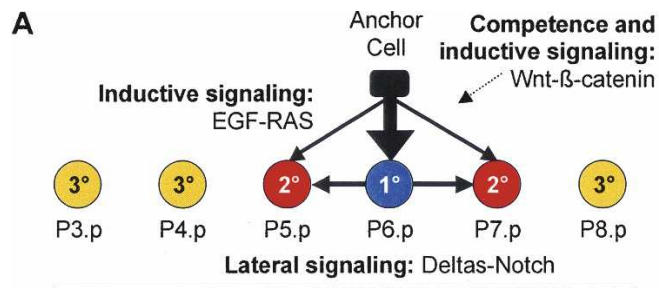

Invariant cell fate pattern

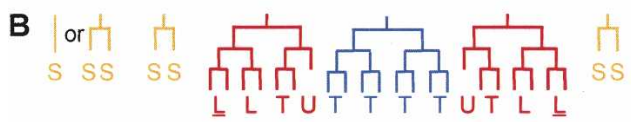

C
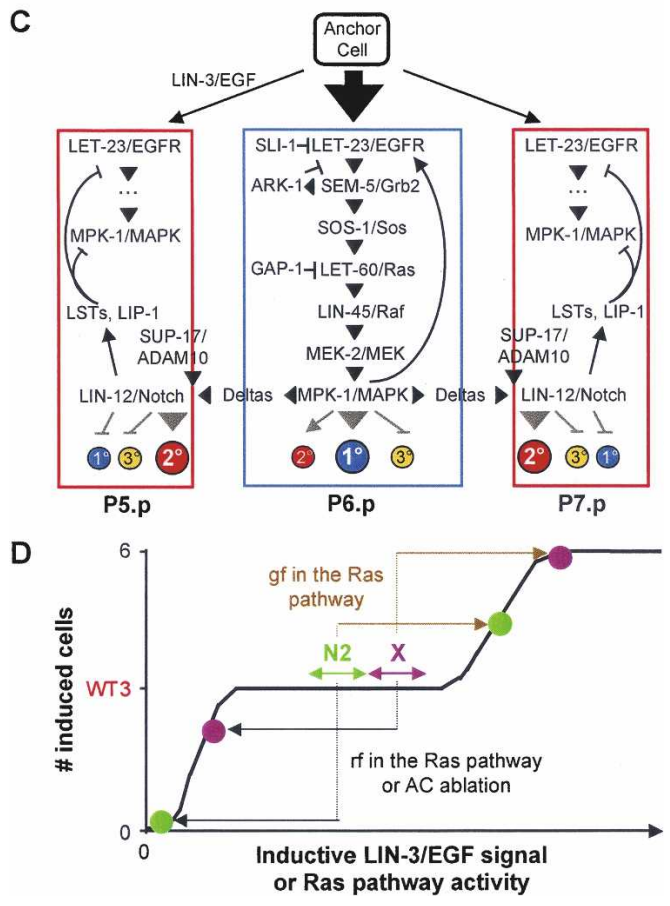
Figure 2. Expressivity of vulval induction defects of Ras, Notch, and Wnt pathway mutations in wild C. elegans backgrounds. We initially chose one mutation per pathway, with an effect on vulval development and no strong lethal effect, and introgressed it into seven wild genetic backgrounds of C. elegans. (A) Ras pathway: let-23(sy1rf)/egfr. The expressivity of this mutation varies significantly among wild $C$. elegans backgrounds (ANOVA, main effect isolate $\mathrm{F}_{6,455}=21.94, P<10^{-4}, n=49$ 70). (B) Notch pathway: sup-17(n316rf)/adam10 (ANOVA, main effect isolate: $\mathrm{F}_{6,441}=5.34, P<10^{-4}$, $n=41-91) . \quad(C)$ Wnt pathway: bar-1(ga801f)/ $\beta$-catenin (ANOVA, main effect isolate: $\left.\mathrm{F}_{6,1657}=20.38, P<10^{-4}, n=187-347\right)$. (rf) Reduction

A Ras pathway let-23(sy1rf)/egfr
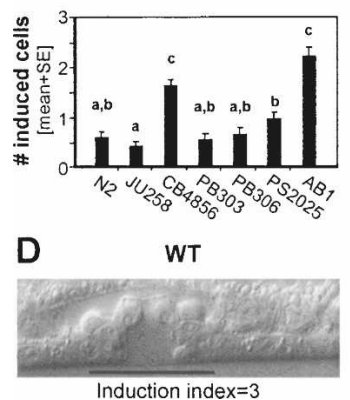

B
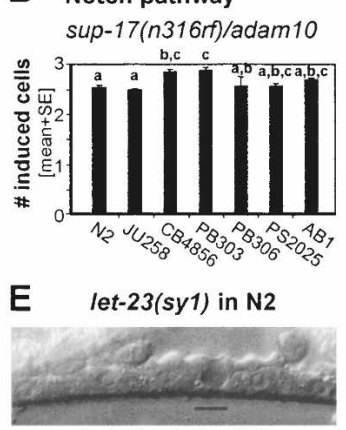

Induction index $=0.5$
C Wnt pathway bar-1(ga80if)/ß-catenin

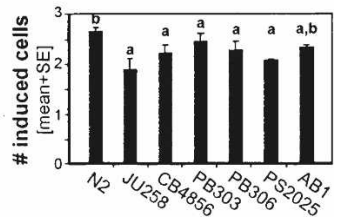

F let-23(sy1) in AB1

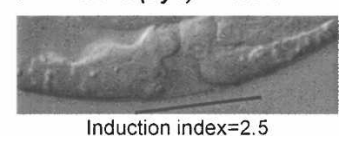
of function; (lf) loss of function. (D) Wild-type vulva at the L4 stage under Nomarski optics. The three induced Pn.p cells give rise to 22 progeny. $(E, F)$ Representative mutant phenotypes of animals carrying the let-23(sy1rf)/egfr mutation in the $\mathrm{N} 2(E)$ and $\mathrm{AB} 1(F)$ backgrounds. Half a Pn.p cell is induced (the posterior daughter of P6.p) in $E$ and 2.5 Pn.p cells in F. Underline indicates vulval tissue. For each mutation, we performed ANOVA tests for the effect of Isolate (the wild genetic background) and of Replicate nested in Isolate. As the effect of Isolate was statistically significant, we next performed post-hoc Tukey's HSD test on Isolate to determine groups of statistical significance (Supplemental Table S4): For each mutation, the mean number of induced cells is significantly different between isolates labeled with different letters $(P<0.05)$ (Supplemental Table S4). Error bars indicate standard error of the mean (SE) over replicates.

removes confounding effects of within-line genetic variation.

In the C. elegans wild isolates, the wild-type pattern consists of three induced Pn.p cells with a $2^{\circ}-1^{\circ}-2^{\circ}$ pattern centered on P6.p, while the other three cells (P3.p, P4.p, and P8.p) remain uninduced. To uncover potential cryptic variation between wild $C$. elegans genetic backgrounds, we first used "invasive" experiments, driving the system out of its buffered range, and scored its output (Fig. 1D). We sensitized the system using two kinds of perturbation: (1) mutations (Gibson and van Helden 1997) and (2) anchor cell ablations (Félix 2007). We next used a "noninvasive" approach through gene reporter transcriptional assays in live worms. Finally, we examined the tissue specificity of the cryptic genetic variation.

We thus uncovered substantial cryptic genetic variation within $C$. elegans impacting the vulval signaling network. The genetic background of different wild isolates affects signaling network activities in a distinct manner, which results in differential temporal dynamics of cell signaling activities and induction. Most observed differences can be explained by differential Ras pathway activities measured in the unperturbed system. Finally, the genetic variation among wild isolates affects the vulva signaling pathways at least partially in a tissuespecific manner.

\section{Results}

Mutations in vulval signaling pathways uncover extensive genetic variation among wild C. elegans isolates

To unravel putative cryptic differences among wild isolates, we first compared the expressivities of mutations that affect the activity of each of the three vulval signaling pathways (Ras, Notch, and Wnt). Each mutation was introgressed by repeated crosses into seven independent wild genetic backgrounds chosen for their molecular or geographic diversity $(\mathrm{N} 2$, the reference laboratory isolate, CB4856, AB1, JU258, PB303, PB306, and PS2025) (Denver et al. 2003; Stewart et al. 2005; Maydan et al. 2007; Dolgin et al. 2008). We scored the phenotypes by counting the number of induced vulval cells; while three cells are induced in the wild-type isolates, this number ranges from 0 to 6 in mutants (Fig. 1).

The effect of Ras pathway mutations differed dramatically among wild isolates (Figs. 2A, 3; Supplemental Table S4) (ANOVA, main effect isolate, $P<0.05$ ). For example, the let-23(sy1)/egfr reduction-of-function (rf) mutation that led to an average of $0.59 \pm 0.04$ S.E. induced cells in the N2 background was strongly suppressed in the AB1 and CB4856 backgrounds to $2.19 \pm 0.11$ cells and $1.62 \pm 0.14$ cells, respectively (Fig. 2A). This suppression required the gonad, since ablating the gonadal precursor cells at the L1 stage resulted in an absence of induction in the strain carrying the let23(sy1) mutation in the CB4856 background $(n=15)$. A mutation in the Notch pathway, sup-17(n316rf)/ adam10, was significantly suppressed, albeit weakly, in the CB4856 and PB303 genetic backgrounds compared with N2 (Fig. 2B). Finally, a null allele of bar-1/ $\beta$-catenin (Wnt pathway) was enhanced in most wild genetic backgrounds compared with $\mathrm{N} 2$ (Fig. $2 \mathrm{C}$ ). bar-1/ $\beta$-catenin is involved (1) in the maintenance of Pn.p cell competence by preventing cell fusion in the L2 stage, and (2) in induction by the anchor cell at the L3 stage. The difference in mean number of induced cells in bar-1/ $\beta$-catenin mutants among wild genetic backgrounds was mainly attributable to variation in the former (1) rather than to the latter (2) (Supplemental Fig. S4). Additionally, bar1(ga80null); let-23(sy1rf) double mutants showed no induced cell in the N2, AB1, and CB4856 backgrounds $(n=20)$ (Supplemental Table S4D), suggesting that suppression of the let-23(sy1) mutation in $\mathrm{AB} 1$ and $\mathrm{CB} 4856$ 


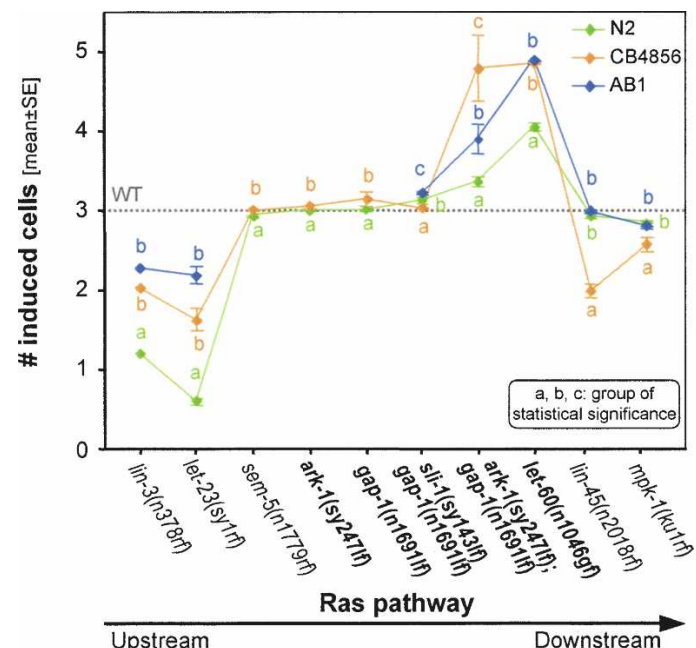

Figure 3. Expressivity of vulval induction defects caused by mutations at different steps along the EGFR-Ras pathway in the N2, CB4856, and AB1 wild genetic backgrounds. The graph shows the number of induced cells ( $Y$-axis, mean and standard error of replicates) for different mutations along the Ras pathway ( $X$-axis, upstream to downstream parts of the pathway from left to right) in three wild genetic backgrounds (colors). Bold lettering indicates mutations that hyperactivate the pathway, while nonbold lettering refers to mutations that underactivate the pathway. See Figure 1C for a schematic drawing of the Ras pathway and its regulators. (rf) Reduction of function (hypomorph); (lf) loss of function (putative null); (gf) gain of function. The 1in-3 and let-23 alleles are tissue specific and strong hypomorphs for the vulva, with no effect on lethality; the other reduction-of-function mutations in positive regulators of the pathway (sem-5, lin-45, and mpk-1 alleles) in addition result in L1 lethality (see Fig. 6) and are milder hypomorphs for the vulva. The other alleles (gain of function in Ras, or loss of function of negative regulators) result in gain of activity in the pathway (more than three cells induced). For each mutation, the mean number of induced cells is significantly different between isolates labeled with different letters (Tukey's HSD test for multiple isolate comparisons and ANOVA main effect Isolate for two isolate comparisons; $P<0.05, n=49-210$ ) (see Fig. 2 legend and Supplemental Table S4 for more explanations).

was not due to other unidentified signaling pathways from the gonad.

We confirmed that the variation of mutation expressivity among genetic backgrounds was not the result of spontaneous mutation during the introgression process using F2 progeny analysis (see the Supplemental Material and Supplemental Figs. S2, S3 for details).

Our results thus clearly showed that the effect of each mutation varied significantly between genetic backgrounds. These findings reveal substantial cryptic variation in the vulval signaling network within C. elegans.

Variation in mutation expressivity suggests that the different genetic backgrounds affect distinct steps of the Ras cascade

Variation between wild genetic backgrounds may occur at one or several steps of the signaling pathways. To localize the effects, we focused on the Ras pathway because of its numerous transduction steps and large number of identified regulators (Fig. 1C) and because of the large variation that was found with let-23(sy1rf)/egfr. We assayed the expressivity of several mutations that affect various steps in the Ras pathway in different wild genetic backgrounds (Supplemental Table S4), concentrating on three isolates, N2, AB1, and CB4856, which showed the largest variation with the let-23(sy1rf)/egfr mutation.

In the $\mathrm{AB} 1$ background, five out of seven tested Ras mutations resulted in a higher number of induced cells than in the N2 background. The isolate effect held both for hypoinduced (induction index <3.0) and hyperinduced (>3.0, bold on Fig. 3) alleles (ANOVA, main effect isolate, $P<0.05$ and post-hoc Tukey's HSD test on isolate, $P=0.05$ ) (Fig. 3). These mutations are not null alleles of the core Ras pathway (which are lethal). Thus, a simple hypothesis for their higher vulval induction level in the $\mathrm{AB} 1$ background is that the activity of the entire Ras pathway is higher in AB1 than in N2, as was confirmed in other experiments (see below).

Only two alleles showed no significant difference between the $\mathrm{N} 2$ and AB1 backgrounds, and both were mild alleles of very small effect (lin-45(n2018rf/raf and mpk1(ku1rf)/mapk) (Fig. 3). The absence of effect of the genetic background on the expressivity of these alleles was consistent with the expectation that, in this robust system, mutants with induction indices that are closer to the wild-type level (three induced cells) are most likely to be the most strongly buffered against genetic background variation (Fig. 1D).

The comparison between N2 and CB4856 revealed a more complex scenario. Seven out of eight mutations or combination of mutations that affected the upstream part of the Ras signaling pathway (Fig. 3) resulted in a higher induction index in CB4856 than in N2 (ANOVA, $P<0.05$ or Tukey's HSD, $P=0.05$ ), while the two mutations that affected the downstream part resulted in the opposite-more induced cells in N2 than in CB4856 (ANOVA, main effect isolate, $P<0.05$ and post-hoc Tukey's HSD test on isolate, $P=0.05)$. To explain the differences between N2 and CB4856, one may hypothesize the occurrence of at least two partially compensating mutations, one affecting the upstream part of the Ras pathway and the other the downstream part.

In summary, the genetic background of each of the three wild isolates affects mutants in the signaling cascade in a distinct manner.

\section{Ablation of the EGF-sending cell uncovers quantitative and qualitative differences in cell fate pattern formation between isolates}

We used a second type of invasive experiment to uncover cryptic differences in vulval development among the three wild isolates N2, CB4856, and AB1. We ablated the anchor cell (the EGF-sending cell) at successive time points during vulval induction. This method allowed us to stop EGF signaling at different developmental stages, 
and thus uncover differences in temporal dynamics of cell fate induction and patterning between isolates (Félix 2007).

In all three isolates, the number of induced cells was very low for early ablations (lethargic L2 stage), increased for intermediate time-point ablations and reached full induction level for late ablations (when the $3^{\circ}$ cells have divided) (Fig. 4A). Yet, the induction started earlier in $\mathrm{AB} 1$ than in $\mathrm{N} 2$, and the wild-type phenotype of three induced cells was reached earlier in AB1 and CB4856 than in N2 (Fig. 4A). In summary, Pn.p cells were induced faster in $\mathrm{AB} 1$ and $\mathrm{CB} 4856$ than in N2 (ANOVA, isolate effect, $P<10^{-4}$ ) (Fig. 4A). These results are con-
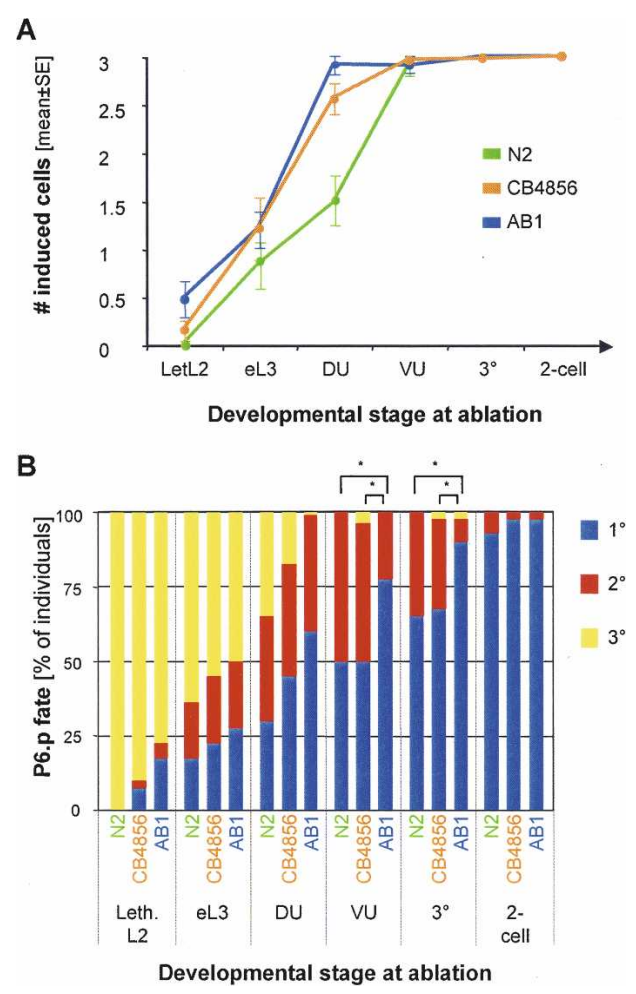

Figure 4. Effect of anchor cell ablation in the N2, CB4856, and AB1 wild isolates. $(A)$ Effect of anchor cell ablation on vulval induction in different $C$. elegans wild isolates over time of ablation. The anchor cell was ablated at different time points ( $X$-axis; $n \geq 20$ individuals per time point, per isolate) and the resulting vulval lineage followed in the early L4 stage. Distributions of the mean number of induced cells differ among isolates (ANOVA, effect isolate: $F_{2,285}=9.96, P<10^{-4}$ ) (Supplemental Table S8). CB4856 and AB1 differ from N2 but not from one another (Tukey's HSD post-hoc test on isolate, $P<0.05$ ). (B) Effect of anchor cell ablation on the cell fate adopted by P6.p in the same experiment. For each time point, the bar shows the proportion of $1^{\circ}$ (blue), $2^{\circ}$ (red), and $3^{\circ}$ (yellow) fates adopted by P6.p in different ablated individuals. $\chi^{2}$ test for the proportion of $1^{\circ}$ versus $2^{\circ}$ cell fates (almost no $3^{\circ}$ cell fate at these time points), $\left(^{\star}\right) P<0.05$. In this test, $\mathrm{AB} 1$ is significantly different from both N2 and CB4856. Developmental stages: (Let. L2) lethargic L2; (eL3) early L3; (DU) Dorsal Uterine precursor cells dividing or divided once; (VU) Ventral Uterine precursor cells dividing or divided once; $\left(3^{\circ}\right) 3^{\circ}$ cells have divided; (two-cell stage) all Pn.p cells have divided once. Cell lineages are shown in Supplemental Tables S5-S7. sistent with the differential effects of Ras pathway mutations.

Examination of cell fate patterns upon anchor cell ablation revealed further qualitative differences. At intermediate time points, P6.p more frequently adopted a $1^{\circ}$ than a $2^{\circ}$ fate in AB1 compared with N2 and CB4856 (Fig. 4B). This suggests that the Ras pathway might overcome the Notch pathway more easily in AB1 than in the other isolates.

Anchor cell ablation also uncovered differential sensitivity of P5.p and P7.p to genetic background. In N2 and AB1, P5.p was more frequently induced than P7.p, while the reverse held true for CB4856 (Supplemental Tables S5-S7). This result matched differential expression of a Ras pathway reporter (see below). Intraspecific variation in induction along the anteroposterior axis may result either from a different position of the anchor cell or from a differential sensitivity of the receiving cells. We did not detect a significant difference in anchor cell position between isolates from the lethargic L 2 to the divided $3^{\circ}$ cell stages (data not shown).

The perturbation methods described above allowed us to uncover cryptic genetic variation among C. elegans wild isolates, suggesting variation in signaling network activity. We next sought to measure variation in molecular pathway parameters in different isolates in the absence of perturbation by analyzing molecular read-outs of the pathways (Félix and Wagner 2008).

\section{Quantitative variation of the Ras pathway output correlates with vulval phenotypes upon perturbation}

To test whether differential Ras pathway activity could explain the differences found among N2, CB4856, and AB1 wild isolates, we performed quantitative assays using transgenic reporters for the pathway outputs. We simultaneously measured Ras pathway output using eg1-17::CFP as a reporter (Yoo et al. 2004) and Notch pathway output using a lip-1::YFP reporter (Fig. 5A,B; Berset et al. 2001). As reported (Yoo et al. 2004), at the lethargic L2 stage, the Ras reporter was expressed in a graded fashion around the anchor cell, with a peak in P6.p, and lower expression in P5.p and P7.p (Fig. 5C). It became restricted to, and increased in intensity in P6.p, the presumptive $1^{\circ}$ cell (Fig. 5C; Yoo et al. 2004). The Notch reporter expression increased over time in P5.p and P7.p, the presumptive $2^{\circ}$ cells, while it remained low in P6.p (Fig. 5C; Berset et al. 2001).

With the Ras pathway reporter, we found significant quantitative and temporal differences among C. elegans isolates (Fig. 5C): At the first time point, Ras reporter intensity was significantly higher in P6.p in $\mathrm{AB} 1$ (983 \pm 120 fluorescence units [f.u.], lethargic L2) and CB4856 (815 \pm 115 f.u.) than in N2 (468 \pm 94 f.u.); this relative difference was maintained until the first Pn.p cell division (Fig. 5C; see Supplemental Table S9 for detailed statistical analysis). Replicates at the lethargic L2 stage with the same and with an independent Ras reporter confirmed this observation (Supplemental Fig. S6; data not shown). Importantly, the higher Ras pathway activity in $\mathrm{AB} 1$ explained the differential effect of Ras 

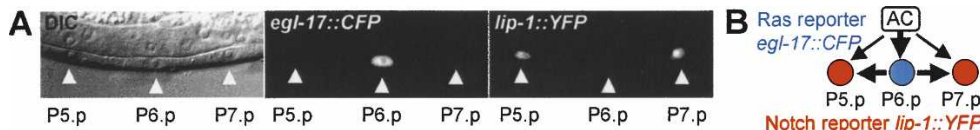

C

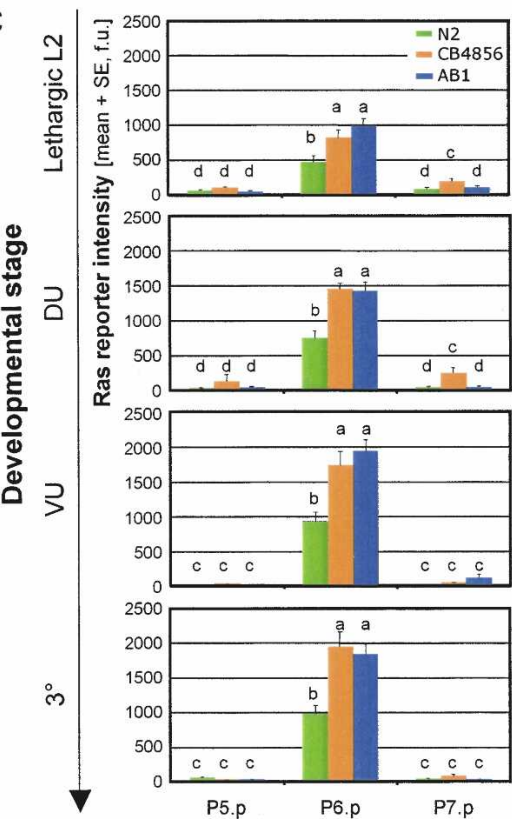

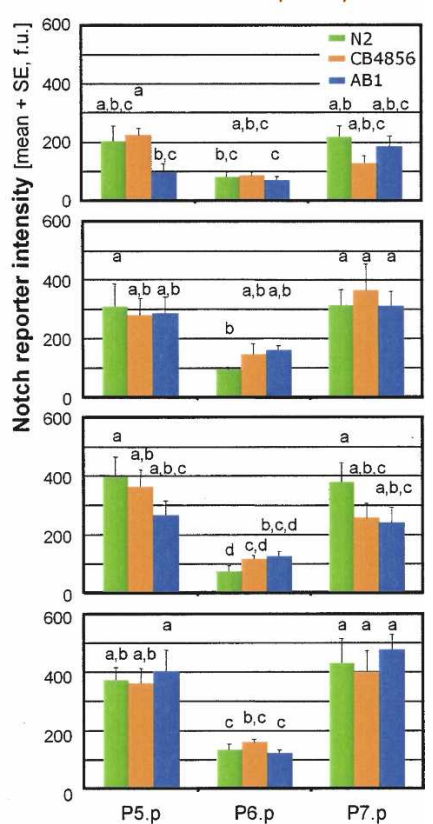

Figure 5. Quantification of downstream transcriptional reporters of the Ras and Notch pathways in the N2, CB4856, and AB1 wild isolates. (A) Micrographs showing the expression pattern of the Ras pathway reporter egl-17::CFP and the Notch pathway reporter lip-1::YFP at the early L3 stage in the same worm. (B) Summary diagram of the expression patterns of egl17::CFP (blue) and lip-1::YFP (red). The egl-17::CFP reporter is repressed over time in P5.p and P7.p, due to lateral Notch signaling. $(C)$ Quantification of the Ras and Notch pathway reporters in three wild isolates. $n=10$ individuals per time point, per isolate. Developmental stages are indicated on the left (see Fig. 4 for nomenclature). (f.u.) (Arbitrary) fluorescence units. We performed ANOVAs for each developmental stage to test for the effects of Isolate (the wild genetic background), Individual nested into Isolate ["Individual (isolate)"], Cell and Isolate by Cell interaction ("Isolate $\times$ Cell") on CFP and on YFP signal intensity. Post-hoc Tukey's HSD $(P<0.05)$ comparisons were then performed on the Isolate $\times$ Cell interaction to determine differences in signal intensity between isolates and cells (P5.p, P6.p, and P7.p). Within one graph, the mean signal intensity is significantly different between cells labeled with different letters. Error bars indicate standard error of the mean (SE) over replicates. For complete ANOVA results, see Supplemental Table S9. pathway mutations, which all resulted in a higher induction index in the AB1 than in the N2 background (Fig. 3). The Ras pathway output was higher in CB4856 than in N2, which was also consistent with a higher induction index of most mutants (Fig. 3). Interestingly, the temporal pattern of expression of the Ras pathway reporter differed in CB4856 from those in N2 and AB1: The signal lasted longer in P5.p and P7.p for CB4856 (Fig. 5C), suggesting that the graded activity of the Ras pathway persisted longer in CB4856.

In the CB4856 isolate, the egl-17::CFP Ras pathway reporter expression was higher in P7.p than in P5.p. This asymmetry matches and explains the anchor cell ablation results in this isolate, where P7.p was more induced than P5.p.

In contrast to the Ras pathway reporter, the Notch pathway reporter showed no significant differences between isolates (Fig. 5C). Since Notch pathway activation in P5.p and P7.p depends on Ras pathway activity in P6.p, this could suggest a compensatory decrease in sensitivity of Notch pathway activation in AB1 and CB4856 compared with N2. An alternative explanation is a buffering of the Notch pathway activation against differences in Ras pathway activity. Nonetheless, we cannot rule out that the Notch reporter was not sensitive enough to uncover differences between isolates.

\section{Tissue-specific effect of wild genetic background on} mutations in the Ras and Wnt pathways

In order to examine whether the observed cryptic genetic variation was affecting the signaling pathway mutations in a tissue-specific manner, we compared the effect of mutations of the Ras and Wnt pathways on vulva development with their effect in other tissues. We selected two other readily scorable phenotypes that are affected by the Ras and Wnt pathways. Both pathways control the specification of the P12 cell: When the activity of either pathway is reduced, P12 can adopt a P11-like cell fate (Jiang and Sternberg 1998). The Ras pathway is also involved in the specification of the excretory duct cell, which is responsible for osmoregulation and required for survival after hatching (Yochem et al. 1997).

We found that the penetrance of mutations varied dramatically with tissues and isolates and the variation was largely uncorrelated across tissues (Fig. 6): For each mutation, at least one isolate belonging to the group of least affected isolates for one tissue belonged to the group of most affected for another. For example, with the lin45(n2018rf)/raf mutation (Fig. 6B; Supplemental Table $\mathrm{S} 12 \mathrm{~B}), \mathrm{AB} 1$ was one of the two most affected isolates for P12 transformation, while it was the least affected for vulval defect and L1 lethality. The opposite held for PB303, while PB306 was mildly affected for all three phenotypes and CB4856 was always amongst the most affected. The interaction term between the genetic background (isolate) and the phenotype was found to be significant for the lin-45(n2018rf) and mpk-1(ku1rf)/mpk mutations (Supplemental Table S13). Thus, the cryptic genetic variation in the vulva intercellular signaling network affects it at least in part in a tissue-specific manner.

\section{Discussion}

Cryptic genetic variation in the vulval signaling network in C. elegans

In this developmental model system, we showed that despite evolutionary stasis in the final cell fate pattern 
Figure 6. Variation in Wnt and Ras pathway mutation effects between tissues among $C$. elegans wild isolates. (A) Effect of bar-1(ga80lf)/ $\beta$-catenin mutation on P12 transformation frequency and vulva defect expressivity in different wild genetic backgrounds (colors). The effect differs between genetic backgrounds for both P12 transformation (G-test, $P<10^{-4}$ ) and vulval defect expressivity (ANOVA, $P<10^{-4}$ ). (B) Effect of lin-45(n2018rf)/ raf on L1 lethality, P12 transformation frequency and vulva defect expressivity. The effect in each of the three tissues varies between genetic backgrounds: $\mathrm{P} 12$ transformation (G-test, $P<10^{-4}$; combined probability for independent G-tests), L1 lethality (G-test, $P<10^{-4}$; combined probability for independent $\mathrm{G}$-tests), and vulval defect expressivity (ANOVA, $P<10^{-4}$ ). (C) Effect of mpk1(ku1rf)/mapk on L1 lethality and vulval defect expressivity. The effect differs between genetic backgrounds for both L1 lethality (G-test, $P<10^{-4}$; combined probability for independent G-tests) and vulval defect expressivity. (ANOVA, $\left.P<10^{-4}\right)$. (D) Effect of sem-5(n1779rf)/grb2 on L1 lethality and vulval defect expressivity. The effect differs between genetic backgrounds for both L1 lethality (G-test, $P<10^{-4}$; combined probability for independent $\mathrm{G}$-tests) and vulval defect expressivity (ANOVA, $P=0.02$ ). (P12 defect) Penetrance of P12 specification defects; (L1 lethality) penetrance of L1 lethality; (vulva) expressivity of vulval defect (vulval induction index). Phenotypic values for each tissue are scaled in each graph by ranking the values from mildest (bottom) to highest (top) penetrance or expressivity (with a minimum scale of 0.2 cell length for vulva data). Error bars indicate SE between replicates, when available. See Supplemental Table S12 for statistical analyses. Lines connect identical genetic backgrounds. Line crossing illustrates the lack of correlation between tissues.

output, genetic variation occurs within the system and is buffered at the level of the output phenotype. Compared with previous studies, the single-cell resolution of the system, its ample description, and the available methods in C. elegans enabled us to explore quantitative variation of the vulval cell signaling network. We first studied quantitative epistatic interactions of a set of signaling network mutations with several wild genetic backgrounds. The matrix of variation of mutation expressivity in the different wild backgrounds is complex and points toward the occurrence of several compensatory network activity changes (Figs. 2, 3; Supplemental Table S4). In addition, we used anchor cell ablations and transcriptional reporter assays to uncover differences among wild isolates. The observed variation in Ras pathway activity among wild isolates matches and explains the differences found by studying quantitative epistatic interactions and by following induction levels in the cell ablation experiments. Finally, the uncovered cryptic genetic variation occurred, at least in part, through tissue-specific evolution and not through correlated evolution affecting pleiotropic signaling pathways in all tissues.

Specifically, the comparison between the N2 and AB1 isolates suggests a simple scenario in which the Ras pathway output (or sensitivity) is increased in the latter compared with the former. This hypothesis is supported by all results with mutations, ablations, and reporters. Using the representation in Figure 1D, the AB1 isolate (like hypothetical isolate X) appears to be located closer to the right end of the plateau than N2. Since Notch pathway activation relies on the Ras pathway (the Ras pathway activates the expression of delta genes that encode Notch receptor ligands) (Fig. 1C), the lack of difference in the downstream activity of the Notch pathway between $\mathrm{AB} 1$ and $\mathrm{N} 2$ may suggest a compensatory decrease in Notch pathway sensitivity in AB1 due to mutation or to buffering of the Notch pathway activation against variation in the Ras pathway activity.

The observed differences between N2 and CB4856 require the postulation of a more complex variation in network activity. The higher expression of a downstream reporter indicates that the net output of the Ras pathway is higher in CB4856 than in N2 (Fig. 5C), most likely as the result of at least two compensatory events along the Ras cascade (Fig. 3). As for AB1, the absence of change in Notch pathway activity may suggest a compensatory decrease in Notch pathway sensitivity in CB4856. In addition, the suppression of reduction-of-function mutations in the Notch pathway [sup-17(n316rf)/adam10 (Fig. 2B); lin-12(n676n930rf)/notch (Supplemental Table S4H)] suggests a lower requirement of the Notch pathway for vulval cell fate patterning in CB4856. On the other hand, detailed analysis of cell fate patterns for $\mathrm{P}(5-7) \cdot \mathrm{p}$ upon anchor cell ablation reveals the occurrence of $2^{\circ} 3^{\circ} 2^{\circ}$ patterns in CB4856, but not in N2 (Supplemental Tables S5, S6). This pattern indicates that the acquisition of a $2^{\circ}$ fate in P5.p and P7.p occurs at a lower Ras pathway ac- 
tivity level than induction of a $1^{\circ}$ fate in P6.p in CB4856 compared with N2. How can we reconcile these observations? Katz et al. (1995) showed that intermediate levels of EGF signaling may trigger a $2^{\circ}$ fate in an isolated Pn.p cell. The long-lasting mild Ras pathway activity in the P5.p and P7.p cells in the wild isolate CB4856 could cooperate with Notch signaling to trigger the $2^{\circ}$ cell fate in these cells. The detailed comparison between N2, CB4856, and AB1 gives experimental support to modeling studies, suggesting that a robust signaling network might enable different wild isolates to adopt different sets of parameters (enzymatic activities, protein levels, affinities, etc.) (von Dassow et al. 2000; Meir et al. 2002; Giurumescu et al. 2006).

In addition, we found spatial differences in cell fate pattern formation. In contrast to $\mathrm{N} 2$ and $\mathrm{AB} 1$, the P7.p cell is more induced than the P5.p cell in CB4856, likely due to the differential Ras pathway activity measured between the two cells. This anteroposterior variation in cell sensitivity to EGF signaling (competence) emphasizes the importance of analyzing any cell signaling network in the context of the whole organism.

We demonstrated differences among C. elegans wild isolates in the penetrance and expressivity of mutations in the Ras pathway, mainly, and also in the Notch and Wnt pathways. These findings have an additional implication relating to the history of vulva genetics: The $C$. elegans vulva genetic screens would likely have given a different mutation spectrum had they been performed in a different C. elegans wild isolate than N2. For example, components of the Wnt pathway that were not identified in the initial screens in the reference isolate N2 (Ferguson and Horvitz 1985; Eisenmann and Kim 2000) might have been easier to identify in the JU258 background, whereas Ras pathway mutants might have been more difficult to isolate in the AB1 background. Similarly, performing the screens on the N2 isolate, but in another environment, might have yielded a different spectrum of mutations (Braendle and Félix 2008). More generally, penetrance of single mutations leading to reduction in induction in some isolates might have been weak, whereas synthetic interactions between Ras and Wnt pathways would have been prevalent.

Anteroposterior variation in sensitivity to LIN-3/EGF is known in the $\mathrm{N} 2$ genetic background to result from the differential expression of the mab-5/hoxB7 gene: $m a b-5 / h o x B 7$ is expressed in a decreasing gradient from the posterior Pn.p cells to P7.p and represses the capacity of P7.p and P8.p to respond to LIN-3/EGF signaling (Clandinin et al. 1997). Lower expression of $\mathrm{mab}-5 /$ hoxB7 in the CB4856 genetic background could lead to an increased competence of P7.p and P8.p. To test this hypothesis, we manipulated Ras pathway activity and mab-5/ hoxb7 expression. In N2 carrying the let-60(n1046)/ras gain-of-function mutation, P8.p induction frequency was $19 \%(n=97)$, a much lower value than that of P4.p $(75 \%)$, while P8.p induction frequency was $60 \%$ in CB4856 ( $n=67)$. Combining the let-60(n1046gf)/ras mutation with mab-5(e1239)/hoxB7 loss of function in N2 led to a much higher induction frequency of P8.p $165 \%$, $n=43$, consistent with Clandinin et al. 1997). Thus, CB4856 appears to display an increased sensitivity (competence) of the posterior Pn.p cells to inductive EGF/Ras signaling, possibly through a decreased activity of $\mathrm{mab}-5$ in these cells.

Using the same experimental paradigm (anchor cell ablation) as in a study of different Caenorhabditis species (Félix 2007), we uncovered qualitatively similar phenotypic variation among $C$. elegans isolates, although within a smaller range than observed at the interspecific level. Thus, evolution of the mechanisms underlying vulva patterning may be attributed to quantitative variation of a network with a single topology, both at the interspecific and intraspecific levels. In addition, the increased posterior Pn.p competence in CB4856 compared with $\mathrm{N} 2$ and $\mathrm{AB} 1$ is similar to that observed in $C$. briggsae (Félix 2007).

Which genetic loci are responsible for the cryptic variation of vulva development in C. elegans? Surveys of wild Drosophila melanogaster populations showed low overall levels of polymorphisms in Ras pathway genes (Gasperini and Gibson 1999). Despite this low polymorphism, association studies for cryptic variation in photoreceptor determination in D. melanogaster uncovered quantitative trait nucleotides at the egfr locus contributing to a small fraction of the genetic variance (Dworkin et al. 2003, 2005). Evolutionary changes in the $C$. elegans vulva system need not occur at loci encoding core components of the Ras, Wnt, or Notch pathways. For example, a different endocytosis rate of the EGFR or the evolution of other tissue-specific regulators could lead to variation in Ras pathway activity. Elucidating the genetic architecture and identifying the involved loci involved in cryptic variation in C. elegans vulva development is now possible using intraspecific crosses between wild genetic backgrounds carrying the same mutant allele (F. Duveau and M.-A. Félix, unpubl.).

\section{What are the evolutionary forces driving cryptic variation?}

Development of a functional vulva (the cell fate pattern output) appears to be under strong stabilizing selection (Braendle and Félix 2008). Here we showed that intraspecific genetic variation affects signaling network activities and dynamics, without change in the final cell fate pattern: The system parameter set evolves within a "neutral" parameter space (i.e., without affecting the system output) (Wagner 2005; Félix and Wagner 2008).

What drives this cryptic evolution? First, exploration of the "neutral" parameter space could be driven by neutral evolutionary dynamics (random drift)—yet bounded by stabilizing selection if reaching the limits of the neutral space. Second, in natural populations or in different laboratory environments, the system is faced with varied ecological contexts that may reveal cryptic variation in the form of deviations in the final vulva pattern: Environmental variation indeed affects mutation penetrance and pathway activities in this system (Braendle and Félix 
2008). These deviations under relevant ecological contexts may be deleterious and impose a selective pressure on the system. Finally, developmental system evolution may be driven through pleiotropic gene action; for example, if levels of activity of the Ras pathway in all tissues were driven to change by selection pressures on one of the many other roles of this pathway. Our results do not rule out some pleiotropic evolution associated with tissue-specific compensation, but point to a great degree of tissue-specific developmental evolution.

\section{Materials and methods}

\section{Wild C. elegans isolates, mutant alleles, and transgenes}

Wild isolates We used seven wild genetic backgrounds that encompass large genetic and geographic variation: N2 (C. elegans reference isolate, Bristol, England), CB4856 (Hawaii), AB1 (Adelaide, Australia), JU258 (Madeira), PB303 (North America), PB306 (North America), and PS2025 (Altadena, California). These strains belong to the two mitochondrial clades defined by Denver et al. (2003). CB4856 and JU258 were isolated on islands and appear highly divergent concerning chemoreceptor gene family evolution (Stewart et al. 2005). These isogenic strains were established by selfing single worms isolated from the wild. The low heterozygosity in wild C. elegans populations suggests that isogenic lines are a good proxy for wild genotypes (Barrière and Félix 2005, 2007).

Mutations We used mutations affecting the three signaling pathways involved in vulva formation. To avoid potential bias, we chose alleles located on different chromosomes and of different genetic and molecular natures. For example, the null alleles belong to the three different types (ochre, amber, and opal). The mutations and corresponding strains are listed in Supplemental Table S1. We sequenced two previously unsequenced alleles, sup-17(n316) and $d p y-20(e 1282)$ (the latter used as a marker in this study).

For sup-17(n316), we amplified two independent PCR products spanning the entire sup-17 coding sequence (primers: oIN9 and oIN10) (Supplemental Table S3A) from MT1789 worms carrying the sup-17(n316) allele. The PCR products were introduced into the pGEMT Easy vector (Promega) and sequenced (primers listed in Supplemental Table S3A). We found a substitution inside the acceptor site of the fifth intron (position 1169 bp of sup-17, WormBase WS186). A guanine is replaced by an adenine (transition).

For $d p y-20($ e1282), we amplified two independent PCR products spanning the whole $d p y-20$ gene (primers: dpy-20_A1 and dpy-20_D2) from WU48 worms carrying the $d p y-20(e 1282)$ allele. These PCR products were directly sequenced (primers listed in Supplemental Table S3A). We identified a point mutation in the antepenultimate exon, which leads to a $354 \mathrm{P}(\mathrm{CCA}) \rightarrow \mathrm{L}$ (CTA) change in the second zinc finger domain (position 2792 of the gene dpy-20; Wormbase WS186).

Transgenes Transgenic lines were established according to standard techniques (Evans 2006) in the N2 background bearing the unc-119(ed3) mutation, using the plasmid unc-119(+) (pDP\#MM016) as a transformation marker.

The lip-1::YFP plasmid (pJM4) was constructed by digesting the pTB10 plasmid (lip-1::GFP, a gift from T. Berset and A.

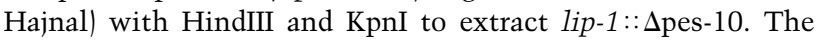

product was ligated into the $2 x N L S:: Y F P$ plasmid (a gift from A Yoo and I. Greenwald) digested with HindIII and NheI.

Integrated transgenic arrays used are $m f I s 4[$ egl-17::YFP, daf6::CFP, unc-119(+)]I, mfIs41[lip-1::NLS-YFP, myo-2::DsRED, unc-119(+)]II, and arIs92[eg1-17::NLS-CFP-1acZ, tax-3::GFP, unc-4(+)]V (a generous gift from A. Yoo and I. Greenwald) (Yoo et al. 2004).

Nonintegrated arrays used are mfEx35[lin-48::GFP, myo-2::DsRED] and mfEx36[1in-48::GFP, myo-2::DsRED]. lin-48::GFP (pTJ1157) is a gift from H. Chamberlin.

Introgression of an allele or transgene of interest into a given C. elegans wild genetic background

No homologous recombination tools are yet available in the different C. elegans wild isolates. We thus introduced each allele by repeated outcrosses (introgression) from a donor strain carrying the allele in the N2 genetic background into the different wild isolate genetic backgrounds (target genome).

Main introgression scheme For recessive alleles, a hermaphrodite with the donor genome carrying the homozygous mutation $(\mathrm{m} / \mathrm{m})$ of interest was crossed to a male of the target genome $(+/+)$ (Supplemental Fig. S1A) (step 1). F1 males $(\mathrm{m} /+)$ were picked and crossed with a hermaphrodite with the target genome $(+/+)$ (step 2). In some introgressions, this step was repeated once (step $\left.2^{\prime}\right)$. Virgin hermaphrodite progeny were isolated $(\mathrm{m} /+$ or $+/+)($ step 3$)$. Their self-progeny were scored for the mutant phenotype $(\mathrm{m} / \mathrm{m})$ (step 4$)$, and grown for one more generation to confirm the phenotype (step 5). The scheme was repeated to yield an average of 10 outcrosses (range six to 15) (see Supplemental Table S2A for the exact values). At the end of the introgression, we produced isogenic lines by propagating single hermaphrodites by selfing for three additional generations.

Modifications to the main introgression scheme The addition of one more outcross per cycle (Supplemental Fig. S1A, step 2') has the advantage of immediately replacing the $\mathrm{X}$ chromosome and of adding one outcrossing event without selection for a phenotype. We applied this modification to the introgression of the following mutations: lin-45(n2018), let-23(sy1), let60(n1046), lin-12(n676n930), mpk-1(ku1), and sem-5(n1779).

The lin-3(n378) allele was introgressed by C. Braendle by repeatedly crossing homozygous lin-3(n378) males with wild hermaphrodites of the target genome. Males were generated by heat-shock at each generation.

The bar-1(ga80)X introgression scheme was modified because hemizygous males carrying this allele are unable to mate. We first generated unc-119(ed3); bar-1(ga80) hermaphrodites. These hermaphrodites were crossed to males, and the cross-progeny identified by its non-Unc phenotype. Hermaphrodites were then selfed once, and Unc animals crossed again to males. We repeated this cycle 10 times. The Unc marker was removed during the last cross.

Sequencing of the mutation: At the end of the introgression, the presence of the mutation was confirmed either by classical sequencing (MWG) or by single-base extension on an ABI 3000avant Prism capillary sequencer (manufacturer's protocol). Primers are listed in Supplemental Table S3B.

Introgression of fluorescent transgenes: Each integrated transgenic array generated in the N2 background was outcrossed 10 times in parallel to the target isolates (N2, CB4856, and AB1), by crossing at each generation the male progeny to wild hermaphrodites. Males carrying the transgenes were identified using fluorescent (dominant) markers expressed in the head. As intro- 
ducing transgenes through the males may affect their expression (Sha and Fire 2005), the same crossing scheme was performed for the three isolates. To get double transgenic lines, a final cross was performed between single introgressed transgenic lines. At the end, hermaphrodites were singled for at least three generations to ensure line isogenicity. See Supplemental Table S2B for a list of strains.

\section{Strain culture and maintenance before an experiment}

We controlled culture conditions and scored all genetic backgrounds in parallel to limit the effect of environmental variation. Worms were grown at $20^{\circ} \mathrm{C}$ unless otherwise stated. For each experiment (or replicate), the worms were freshly thawed and grown for four to six generations on standard 55-mm NGM plates, all poured the same day and all seeded the same day, with a single batch of food (Escherichia coli OP50). The strains were usually bleached at generation 3-4 to remove any contamination. The analyses were carried out within 1-2 d [except for the analysis of bar-1(ga80lf) replicate 1, for which a few individuals from each strain were chosen randomly in succession over a week].

\section{Statistical analyses}

Statistical tests were performed using the JMP7.0 and SPSS11.0 softwares for Macintosh. For parametric tests, data were transformed using Box-Cox transformations to meet the assumptions of ANOVA procedures (homogeneity of variances and normal distributions of residuals). For post-hoc comparisons, Tukey's HSD procedure was used. We analyzed data for which ANOVA assumptions could not be met using nonparametric MannWhitney U-test and Kruskal-Wallis F-test. For comparison of proportional data (percentage of cell fate specification defects, penetrance of mutations), we used $\chi^{2}$ tests and G-tests of independence (Sokal and Rohlf 1994). We applied the Williams' correction when required (less than five individuals in one category). Fisher's correction for independent tests was used to take into account replicates. To compare the effect of a mutation among different tissues and genetic backgrounds, we performed a rank test (Scheirer-Ray-Hare extension of the KruskalWallis test) (Sokal and Rohlf 1994) on the normalized mean phenotype for each tissue. Error bars stand for one standard error of the mean (SE).

\section{Analysis of the phenotypes}

Vulva The vulva phenotype was scored at the L4 stage using Nomarski optics as described previously (Sternberg and Horvitz 1986). The number of induced Pn.p cells (induction index) is deduced from cell lineage output (Fig. 1B). The wild-type phenotype is three induced cells (Fig. 1A) and all seven wild isolates show this precise phenotype under normal conditions. Upon perturbation, the number of induced cells ranges from zero to six cells. Frequently in mutants, only one daughter of a Pn.p cell adopts a vulval fate, while the other adopts a nonvulval fate (fusion to hyp7). In such a case, the Pn.p cell will contribute 0.5 to the induction index (see Fig. 2D,E for examples).

Excretory duct cell The excretory duct cell is necessary for worm osmoregulation. Reduction of function in the Ras pathway can lead to its absence, causing death of the L1 larva after hatching, with a characteristic rod-like phenotype (Yochem et al. 1997). The penetrance of L1 lethality for Ras pathway reduction-of-function mutations was scored as follows: Ten to 20 adult hermaphrodites were placed on fresh culture plates and allowed to lay eggs for a few hours (so as to obtain 50-100 eggs). Laid eggs were counted after removing the adults, and incubated for $2 \mathrm{~d}$ at $20^{\circ} \mathrm{C}$ or $25^{\circ} \mathrm{C}$. L1 lethality was deduced from the number of living larvae and dead embryos.

Hyperactivation of the Ras pathway can lead to the formation of a second excretory duct cell. For gain-of-function mutations in the Ras pathway, the number of excretory duct cells was scored in parallel cultures at the L4 larval stage under Nomarski optics (Yochem et al. 1997). The second duct cell was sometimes located more ventrally. To confirm its duct cell fate, we used lin-48:: GFP, a duct cell reporter (Johnson et al. 2001).

P12 cell fate specification P12 specification depends on the activity of both Ras and Wnt pathways (Jiang and Sternberg 1998). Reduction of function in either pathway may lead P12 to adopt a P11-like cell fate. P12 specification was scored by counting the number of P12.pa-like and P11.p-like cells at the L4 larval stage using Nomarski optics, as described previously (Aroian and Sternberg 1991).

\section{Cell lineage and cell ablation}

The anchor cell was ablated using a laser microbeam (Photonics Instruments) as described (Félix 2007). We selected worms from asynchronous $25^{\circ} \mathrm{C}$ cultures and staged them under Nomarski optics based on morphological traits and cell division pattern (Félix 2007).

We distinguished the lethargus L2 stage by the closed mouth and the size of the worm. The early L3 (eL3) stage corresponds to the time between the molt and DU cell division. "DU" are Dorsal Uterine precursors; "VU" are Ventral Uterine precursors (Kimble and Hirsh 1979). The "DU stage" corresponds to the stage when DU cells have divided once, but VU cells have not. The "VU stage" corresponds to the time when the VU cells are dividing or have divided, but no Pn.p has yet divided. The " $3^{\circ}$ stage" corresponds to the time when $3^{\circ}$ cells have divided, but not $\mathrm{P}(5-7) . \mathrm{p}$. The "two-cell stage" corresponds to the time when all competent Pn.p cells have divided once.

After ablation, worms were recovered and grown at $25^{\circ} \mathrm{C}$. Cell lineage was inferred from observations at the lethargus L3 stage and about $2 \mathrm{~h}$ later under Nomarski optics (100× objective), as described (Félix 2007). Cell lineage nomenclature is given in Figure 1 and Supplemental Table S5. S or s are scored as $3^{\circ}$ fates. LL, LL, and vulval sublineages with a cell adhering to the cuticle were scored as external $2^{\circ}$. TU was scored as an internal $2^{\circ}$ fate and TT as a half $1^{\circ}$. O was interpreted as equivalent to a $\mathrm{T}$. $\mathrm{D}$ was interpreted depending on the lineage context (for example, TDDT, $1^{\circ}$; LDTU, $2^{\circ}$. See Félix (2007) for discussion on ambiguous lineage cases.

\section{Fluorescence analysis}

Worms from an asynchronous culture were mounted on pads of $5 \%$ noble agar in M9 (Shaham 2006), to which $10 \mathrm{mM}$ sodium azide was added. Individuals from the different wild genetic backgrounds of interest were assayed in parallel. Staging was performed by morphological criteria as above. Nomarski and fluorescent images were acquired on a Zeiss AxioImager M1 microscope (100x objective) with a Photometrics CoolSnap ES CCD camera driven by the Metaview 6.3r7 software. For quantification of YFP and CFP signals, all images were acquired using the same materials and settings for a given transgene. The focal plane was chosen to cross the center of the Pn.p cell nuclei. The signal intensity was quantified using the ImageJ1.37 software (Abramoff et al. 2004). The mean background intensity measured outside the worm was subtracted from each picture. 
For nuclearly localized reporters (egl-17::CFP and lip-1::YFP), we measured the mean intensity in the brightest subregion of fixed size (disk of 10-pixel diameter) in the Pn.p cell nucleus. This method gave highly similar results and was faster than measuring signal intensity over the whole nucleus (defined by hand using the polygonal tool of ImageJ on the Nomarski picture): The correlation coefficient between both methods was 0.99 for the CFP reporter and 0.98 for the YFP reporter $\mid n=30$ individuals for each reporter at the L2 stage).

For the tail cells (Supplemental Fig. S5A), the mean intensity was measured over the whole nucleus (defined by hand using the polygonal tool of ImageJ on the Nomarski picture). Note that the egl-17 and lip-1 promoters have only been shown to respond to the Ras and Notch pathways, respectively, in the vulval precursor cells, but not in other tissues.

For the cytoplasmic reporter eg1-17::YFP, the signal was diffuse over the whole cell and heterogeneous. We used the threshold function of ImageJ on the YFP picture to define each Pn.p cell. We measured the integrated density over the area hence defined. Note that the mean and the maximum signal intensity also gave very similar results (correlation coefficient $>0.9$ for any correlation).

\section{Acknowledgments}

We thank the Caenorhabditis Genetics Center (funded by NIHNCRR), A. Yoo, I. Greenwald, T. Berset, A. Hajnal, H. Chamberlin, N. Hopper, and P. Sternberg for strains and plasmids, and A. Keyte for performing some introgressions. We acknowledge the IJM imaging facility for technical assistance with microscope settings. We thank C. Braendle for the lin-3 introgression results and his help with statistical analyses. We are grateful to G. Gibson for inspiration and to C. Braendle, F. Hospital, V. Orgogozo, and M. Sundaram for discussions. We thank C. Braendle, M. Delattre, I. Dworkin, V. Orgogozo, J.-B. Pénigault, and J. Rothman for comments on versions of the manuscript. This work was funded by the Centre National de la Recherche Scientifique and grants from the Human Frontier Science Program (RGP0016/2001), the Association pour la Recherche sur le Cancer (no. 3749), and the Agence Nationale de la Recherche (ANR-05-BLAN-0231-01). J.M. was supported by a Ph.D. fellowship of the French Ministry of Research and the ARC. F.D. is supported by a Ph.D. fellowship of the French Ministry of Research.

\section{References}

Abramoff, M.D., Magelhaes, P.J., and Ram, S.J. 2004. Image Processing with ImageJ. Biophotonics Int'1. 11: 36-42.

Alon, U., Surette, M.G., Barkai, N., and Leibler, S. 1999. Robustness in bacterial chemotaxis. Nature 397: 168-171.

Aroian, R.V. and Sternberg, P.W. 1991. Multiple functions of let-23, a Caenorhabditis elegans receptor tyrosine kinase gene required for vulval induction. Genetics 128: 251-267.

Barkai, N. and Leibler, S. 1997. Robustness in simple biochemical networks. Nature 387: 913-917.

Barrière, A. and Félix, M.A. 2005. High local genetic diversity and low outcrossing rate in Caenorhabditis elegans natural populations. Curr. Biol. 15: 1176-1184.

Barrière, A. and Félix, M.A. 2007. Temporal dynamics and linkage disequilibrium in natural Caenorhabditis elegans populations. Genetics 176: 999-1011.

Berset, T., Hoier, E.F., Battu, G., Canevascini, S., and Hajnal, A. 2001. Notch inhibition of RAS signaling through MAP ki- nase phosphatase LIP-1 during C. elegans vulval development. Science 291: 1055-1058.

Braendle, C. and Félix, M.-A. 2008. Plasticity and errors of a robust developmental system in different environments. Dev. Cell (in press).

Braendle, C., Milloz, J., and Félix, M.A. 2008. Mechanisms and evolution of environmental responses in Caenorhabditis elegans. Curr. Top. Dev. Biol. 80: 171-207.

Clandinin, T.R., Katz, W.S., and Sternberg, P.W. 1997. Caenorhabditis elegans HOM-C genes regulate the response of vulval precursor cells to inductive signal. Dev. Biol. 182: 150-161.

Delattre, M. and Félix, M.A. 2001. Polymorphism and evolution of vulval precursor cell lineages within two nematode genera, Caenorhabditis and Oscheius. Curr. Biol. 11: 631643.

Denver, D.R., Morris, K., and Thomas, W.K. 2003. Phylogenetics in Caenorhabditis elegans: An analysis of divergence and outcrossing. Mol. Biol. Evol. 20: 393-400.

de Visser, J.A., Hermisson, J., Wagner, G.P., Ancel Meyers, L., Bagheri-Chaichian, H., Blanchard, J.L., Chao, L., Cheverud, J.M., Elena, S.F., Fontana, W., et al. 2003. Perspective: Evolution and detection of genetic robustness. Evolution Int. J. Org. Evolution 57: 1959-1972.

Dolgin, E.S., Félix, M.A., and Cutter, A.D. 2008. Hakuna Nematoda: Genetic and phenotypic diversity in African isolates of Caenorhabditis elegans and C. briggsae. Heredity 100: 304315

Dworkin, I., Palsson, A., Birdsall, K., and Gibson, G. 2003. Evidence that Egfr contributes to cryptic genetic variation for photoreceptor determination in natural populations of Drosophila melanogaster. Curr. Biol. 13: 1888-1893.

Dworkin, I., Palsson, A., and Gibson, G. 2005. Replication of an Egfr-wing shape association in a wild-caught cohort of Drosophila melanogaster. Genetics 169: 2115-2125.

Eisenmann, D.M. and Kim, S.K. 2000. Protruding vulva mutants identify novel loci and Wnt signaling factors that function during Caenorhabditis elegans vulva development. Genetics 156: 1097-1116.

Eisenmann, D.M., Maloof, J.N., Simske, J.S., Kenyon, C., and Kim, S.K. 1998. The $\beta$-catenin homolog BAR-1 and LET-60 Ras coordinately regulate the Hox gene lin-39 during Caenorhabditis elegans vulval development. Development 125: 3667-3680.

Eldar, A., Dorfman, R., Weiss, D., Ashe, H., Shilo, B.Z., and Barkai, N. 2002. Robustness of the BMP morphogen gradient in Drosophila embryonic patterning. Nature 419: 304-308.

Eldar, A., Rosin, D., Shilo, B.Z., and Barkai, N. 2003. Self-enhanced ligand degradation underlies robustness of morphogen gradients. Dev. Cell 5: 635-646.

Evans, T.C. 2006. Transformation and microinjection. In WormBook (ed. The C. elegans Research Community), WormBook. doi: 10.1895/wormbook.1.108.1, http://www. wormbook.org.

Félix, M.A. 1999. Evolution of developmental mechanisms in nematodes. J. Exp. Zool. 285: 3-18.

Félix, M.A. 2007. Cryptic quantitative evolution of the vulva intercellular signaling network in Caenorhabditis. Curr. Biol. 17: 103-114.

Félix, M.A. and Wagner, A. 2008. Robustness and evolution: Concepts, insights and challenges from a developmental model system. Heredity 100: 132-140.

Félix, M.A., De Ley, P., Sommer, R.J., Frisse, L., Nadler, S.A., Thomas, W.K., Vanfleteren, J., and Sternberg, P.W. 2000 Evolution of vulva development in the Cephalobina (Nematoda). Dev. Biol. 221: 68-86. 
Ferguson, E.L. and Horvitz, H.R. 1985. Identification and characterization of 22 genes that affect the vulval cell lineages of the nematode Caenorhabditis elegans. Genetics 110: 17-72.

Flatt, T. 2005. The evolutionary genetics of canalization. Q. Rev. Biol. 80: 287-316.

Gasperini, R. and Gibson, G. 1999. Absence of protein polymorphism in the Ras genes of Drosophila melanogaster. J. Mol. Evol. 49: 583-590.

Gibson, G. and Dworkin, I. 2004. Uncovering cryptic genetic variation. Nat. Rev. Genet. 5: 681-690.

Gibson, G. and van Helden, S. 1997. Is function of the Drosophila homeotic gene Ultrabithorax canalized? Genetics 147: 1155-1168.

Gibson, G. and Wagner, G. 2000. Canalization in evolutionary genetics: A stabilizing theory? Bioessays 22: 372-380.

Gibson, G., Wemple, M., and van Helden, S. 1999. Potential variance affecting homeotic Ultrabithorax and Antennapedia phenotypes in Drosophila melanogaster. Genetics 151: 1081-1091.

Giurumescu, C.A., Sternberg, P.W., and Asthagiri, A.R. 2006. Intercellular coupling amplifies fate segregation during Caenorhabditis elegans vulval development. Proc. Natl. Acad. Sci. 103: 1331-1336.

Green, J.L., Inoue, T., and Sternberg, P.W. 2008. Opposing Wnt pathways orient cell polarity during organogenesis. Cell 134: 646-656.

Gregor, T., Tank, D.W., Wieschaus, E.F., and Bialek, W. 2007. Probing the limits to positional information. Cell 130: 153164.

Jiang, L.I. and Sternberg, P.W. 1998. Interactions of EGF, Wnt and HOM-C genes specify the P12 neuroectoblast fate in $C$. elegans. Development 125: 2337-2347.

Johnson, A.D., Fitzsimmons, D., Hagman, J., and Chamberlin, H.M. 2001. EGL-38 Pax regulates the ovo-related gene lin-48 during Caenorhabditis elegans organ development. Development 128: 2857-2865.

Katz, W.S., Hill, R.J., Clandinin, T.R., and Sternberg, P.W. 1995. Different levels of the C. elegans growth factor LIN-3 promote distinct vulval precursor fates. Cell 82: 297-307.

Kimble, J. and Hirsh, D. 1979. The postembryonic cell lineages of the hermaphrodite and male gonads in Caenorhabditis elegans. Dev. Biol. 70: 396-417.

Kiontke, K., Barrière, A., Kolotuev, I., Podbilewicz, B., Sommer, R., Fitch, D.H., and Félix, M.A. 2007. Trends, stasis, and drift in the evolution of nematode vulva development. Curr. Biol. 17: 1925-1937.

Kirouac, M. and Sternberg, P.W. 2003. Cis-regulatory control of three cell fate-specific genes in vulval organogenesis of Caenorhabditis elegans and C. briggsae. Dev. Biol. 257: 85-103.

Maydan, J.S., Flibotte, S., Edgley, M.L., Lau, J., Selzer, R.R., Richmond, T.A., Pofahl, N.J., Thomas, J.H., and Moerman, D.G. 2007. Efficient high-resolution deletion discovery in Caenorhabditis elegans by array comparative genomic hybridization. Genome Res. 17: 337-347.

Meir, E., von Dassow, G., Munro, E., and Odell, G.M. 2002. Robustness, flexibility, and the role of lateral inhibition in the neurogenic network. Curr. Biol. 12: 778-786.

Sha, K. and Fire, A. 2005. Imprinting capacity of gamete lineages in Caenorhabditis elegans. Genetics 170: 1633-1652.

Shaham, S. 2006. Methods in cell biology. In WormBook (ed. The C. elegans Research Communityl, WormBook. doi: 10.1895/wormbook.1.49.1, http://www.wormbook.org.

Sokal, R.R. and Rohlf, F.J. 1994. Biometry: The principles and practice of statistics in biological research. W.H. Freeman and Co., New York.

Sommer, R.J. 2005. Evolution of development in nematodes re- lated to C. elegans. In WormBook (ed. The C. elegans Research Community), WormBook. doi: 10.1895/wormbook.1.46.1, http://www.wormbook.org.

Sternberg, P.W. 2005. Vulval development. In WormBook (ed. The C. elegans Research Community), WormBook. doi: 10.1895/wormbook.1.6.1, http://www.wormbook.org.

Sternberg, P.W. and Horvitz, H.R. 1986. Pattern formation during vulval development in C. elegans. Cell 44: 761-772.

Stewart, M.K., Clark, N.L., Merrihew, G., Galloway, E.M., and Thomas, J.H. 2005. High genetic diversity in the chemoreceptor superfamily of Caenorhabditis elegans. Genetics 169: 1985-1996.

von Dassow, G., Meir, E., Munro, E.M., and Odell, G.M. 2000. The segment polarity network is a robust developmental module. Nature 406: 188-192.

Wagner, A. 2005. Robustness and evolvability in living systems. Princeton University Press, Princeton, NJ.

Yochem, J., Sundaram, M., and Han, M. 1997. Ras is required for a limited number of cell fates and not for general proliferation in Caenorhabditis elegans. Mol. Cell. Biol. 17: 27162722.

Yoo, A.S., Bais, C., and Greenwald, I. 2004. Crosstalk between the EGFR and LIN-12/Notch pathways in C. elegans vulval development. Science 303: 663-666.

Zauner, H. and Sommer, R.J. 2007. Evolution of robustness in the signaling network of Pristionchus vulva development. Proc. Nat1. Acad. Sci. 104: 10086-10091. 


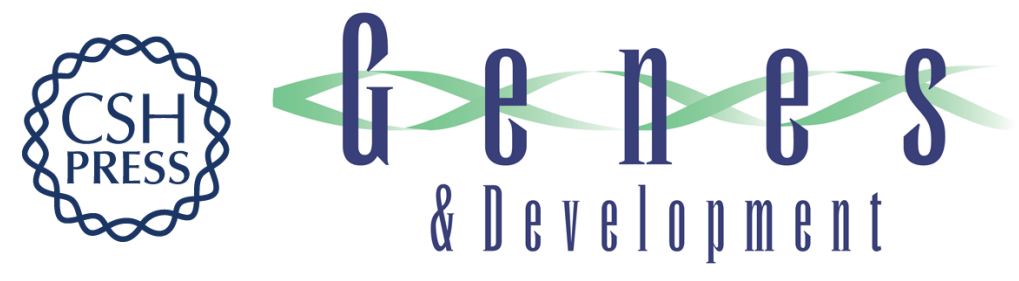

\section{Intraspecific evolution of the intercellular signaling network underlying a robust developmental system}

Josselin Milloz, Fabien Duveau, Isabelle Nuez, et al.

Genes Dev. 2008, 22:

Access the most recent version at doi:10.1101/gad.495308

Supplemental http://genesdev.cshlp.org/content/suppl/2008/11/05/22.21.3064.DC1
Material

References This article cites 48 articles, 18 of which can be accessed free at:

http://genesdev.cshlp.org/content/22/21/3064.full.html\#ref-list-1

License

Email Alerting Receive free email alerts when new articles cite this article - sign up in the box at the top

Service

right corner of the article or click here.

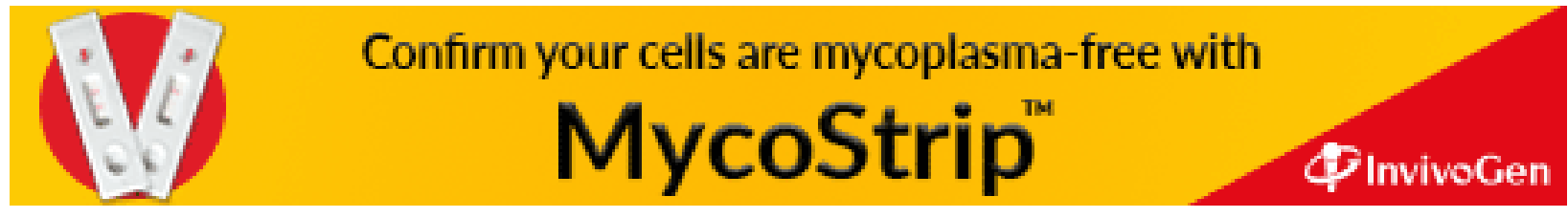

